\title{
Silicon Photonics for Optical Fiber Communication Applications
}

\author{
D. Vermeulen ${ }^{1}$, G. Roelkens ${ }^{2}$, D. Van Thourhout ${ }^{2}$, C.R. Doerr ${ }^{1}$ \\ ${ }^{1}$ Acacia Communications Inc., Three Clock Tower Place, Suite 210, Maynard, MA 01754, USA \\ 2 Photonics Research Group, Department of Information Technology, \\ Ghent University - imec, B-9000 Ghent, Belgium
}

\begin{abstract}
We will give an overview of the state-of-the-art in Silicon Photonics advancements focusing on the optical power budget and polarization requirements for applications in optical fiber communications.
\end{abstract}

\section{Summary}

Photonic integration, in the sense that the light is fully controlled using on-chip waveguides, has opened up a whole new dimension of photonic components and the ability to squeeze complex circuits onto a single chip. However, the early cases of photonic integration successes were single photonic components such as photodetectors, modulators and wavelength demultiplexers having a superior performance over their discrete counterparts. This increased performance is mainly achieved by tuning the integration platform according to the specific component needs. A couple of examples are the III-V materials for lasers and photodetectors, silica glass for passive optical filters and Lithium Niobate for modulators. Photonic systems quickly became an assembly of fiber based components and single integrated components, merging the best of all worlds. But, as research and technology in integrated photonics advances, the chance that these multi-systems dominate the photonics landscape is decreasing dramatically. Factors such as size, cost and power consumption are becoming more important every day and are the main driving force behind the search for the holy grail of complete photonic integration.

The applications of integrated photonic circuits are numerous and range from active cables to sensing and free space applications. However, although the integrated photonics technology has the potential to become a major player in all these fields, it has to become the solution for future telecom applications due to the inexistence of any alternative to meet future size and complexity requirements. The main challenge in all of this is to develop this complete photonic integration platform that has the potential to meet the required specifications. We will evaluate Silicon Photonics candidature as integration platform for telecom applications.

The main reason people started looking into silicon as photonic material (at telecom wavelengths) is because of the already developed silicon industry for micro-electronics. Silicon technology has been massively optimized during several decades now and has reached a point that it became impossible for other platforms to even scratch the uniformity, cost-yield ratio and quality of silicon processed integrated circuits. Combining Ge with silicon has been proven to be quite straightforward and paved the way for high quality photodetectors [1]. Furthermore, using doped waveguides high-speed modulation can be achieved [2]. However, one major problem is the integration of light sources due to the indirect bandgap of silicon. Several solutions are developed based on the integration of III-V material on the silicon platform by means of hybrid or heterogeneous integration [3]. None of them could be called elegant but until the Ge laser, which has recently showed to be very promising [4], or an other truly CMOS-compatible laser delivers, these solutions are second to none. Another alternative approach could be to completely separate the lasers and the rest of circuit using a co-packaged III-V and Silicon Photonics chip but at the cost of increased packaging costs.

Putting the laser issue aside as this is at the moment a very application dependent problem and solutions come in many flavors, one big general problem that Silicon Photonics is fighting with are the total circuit losses and specifically the fiber-to-chip coupling losses. Furthermore, due to the high birefringence of the silicon waveguides, both orthogonal polarizations are treated preferably independently using a polarization diversity circuit. In order to have full benefit from the cost-yield ratio of the Silicon Photonics platform, low-cost chip testing is a must and this can only be provided if surface couplers such as grating couplers are used ([5], [6]), thereby enabling wafer-scale testing and thus pre-packaging die selection. We will take the coupling problem as a starting point for discussing silicon photonic integration for applications such as Fiber-To-The-Home (FTTH) Point-To-Point (PTP) [7] and coherent optical 
transceivers [8].

\section{References and links}

1. L. Vivien, J. Osmond, J.-M. Fédéli, D. Marris-Morini, P. Crozat, J.-F. Damlencourt, E. Cassan, Y. Lecunff, and S. Laval, "42 GHz p.i.n Germanium photodetector integrated in a silicon-on-insulator waveguide." Optics express, vol. 17, no. 8, pp. 6252-7, 2009.

2. F. Y. Gardes, G. T. Reed, a. P. Knights, and G. Mashanovich, "Evolution of optical modulation using majority carrier plasma dispersion effect in SOI," Proceedings of SPIE, vol. 6898, pp. 68 980C-68 980C-10, 2008.

3. G. Roelkens, J. V. Campenhout, J. Brouckaert, D. V. Thourhout, R. Baets, P. R. Romeo, P. Regreny, A. Kazmierczak, C. Seassal, X. Letartre, G. Hollinger, J. M. Fedeli, and L. D. Cioccio, "III-V/Si photonics by die-to-wafer bonding," Materials Today, vol. 10, no. 7, pp. 36-43, 2007.

4. R. E. Camacho-Aguilera, Y. Cai, N. Patel, J. T. Bessette, M. Romagnoli, L. C. Kimerling, and J. Michel, "An electrically pumped germanium laser." Optics express, vol. 20, no. 10, pp. 11 316-20, 2012.

5. D. Taillaert, P. Bienstman, and R. Baets, "Compact efficient broadband grating coupler for silicon-on-insulator waveguides." Optics letters, vol. 29, no. 23, pp. 2749-51, 2004.

6. A. Mekis, S. Gloeckner, G. Masini, A. Narasimha, T. Pinguet, S. Sahni, and P. Dobbelaere, "A Grating-Coupler-Enabled CMOS Photonics Platform," Selected Topics in Quantum Electronics, IEEE Journal of, no. 99, pp. 1-12, 2010.

7. D. Vermeulen and G. Roelkens, "Silicon-on-insulator nanophotonic waveguide circuit for fiber-to-the-home transceivers," , 2008. ECOC 2008., vol. 2, no. September, pp. 1-2, 2008.

8. C. Doerr, L. Buhl, Y. Baeyens, R. Aroca, S. Chandrasekhar, X. Liu, L. Chen, and Y. Chen, "Packaged monolithic silicon 112-Gb/s coherent receiver," Photonics Technology Letters, IEEE, vol. 23, no. 99, pp. 1-1, 2011. 